\title{
THE PRIMITIVE LIFTING PROBLEM IN THE EQUIVALENCE PROBLEM FOR TRANSITIVE PSEUDOGROUP STRUCTURES:
}

\section{A COUNTEREXAMPLE}

\author{
BY
}

\author{
PIERRE MOLINO
}

\begin{abstract}
A transitive Lie pseudogroup $\Gamma_{M}$ on $M$ is a primitive extension of $\Gamma_{N}$ if $\Gamma_{N}$ is the quotient of $\Gamma_{M}$ by an invariant fibration $\pi: M \rightarrow$ $N$ and if the pseudogroup induced by $\Gamma_{M}$ on the fiber of $\pi$ is primitive. In the present paper an example of this situation is given with the following property (counterexample to the primitive lifting property): the equivalence theorem is true for almost- $\Gamma_{N^{\text {structures }}}$ but false for almost- $\Gamma_{M}$ structures.
\end{abstract}

1. We shall consider the following situation: Let $\pi: M \rightarrow N$ be a fibration of smooth manifolds, $\Gamma_{M}$ a transitive Lie pseudogroup on $M$ respecting the fibration $\pi$. It is not always true that a quotient pseudogroup on $N$ can be defined, but by projecting the equations of $\Gamma_{M}$ (that is to say the associated structures) we obtain the equations of a transitive Lie pseudogroup $\Gamma_{N}$ on $N . \Gamma_{N}$ will be referred to as the pseudogroup defined by passing to the quotient. In the study of the equivalence problem for almost- $\Gamma_{M^{-}}$-structures (see [1], [3], [4]), it is a standard method to use such quotients. If the equivalence theorem is true

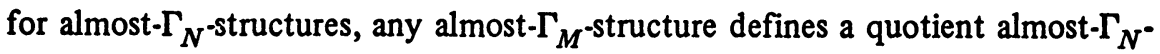
structure, and the given equivalence problem reduces to a "lifting problem" from an equivalence for the quotient structure to an equivalence for the given structure.

Let $K$ be a fiber of $\pi, \Gamma_{K}$ the family of restrictions to $K$ of those transformations in $\Gamma_{M}$ which map $K$ into $K$. We assume here that $\Gamma_{K}$ is a flat irreducible transitive Lie pseudogroup on $K$. Then $\Gamma_{K}$ is either an affine pseudogroup $\left(\Gamma_{M}\right.$ is an "affine extension" of $\left.\Gamma_{N}\right)$ or a primitive pseudogroup $\left(\Gamma_{M}\right.$ is a "primitive extension" of $\Gamma_{N}$ ).

The purpose of this paper is to give an example where $\Gamma_{M}$ is a primitive extension of $\Gamma_{N}$ and the equivalence theorem is true for $\Gamma_{N}$ but false for $\Gamma_{M}$. In the terminology of A. Pollack (see [4]), the "primitive lifting theorem for $\Gamma_{M} \rightarrow \Gamma_{N}$ " is not true.

Received by the editors July 25, 1975.

AMS (MOS) subject classifications (1970). Primary 58A15; Secondary 53C10, 57D55.

Key words and phrases. Equivalence problem, almost- $\Gamma$-structures, primitive Lie pseudogroups, Lewy counterexample, equivalence theorem, flat pseudogroups, primitive lifting theorem. 
In the paper just mentioned, A. Pollack asserts that, for $\Gamma_{M}$ and $\Gamma_{N}$ flat, "the primitive lifting theorem can be established with little difficulty", while "the affine lifting theorem requires deep results from partial differential equation theory" (essentially Ehrenpreis-Malgrange's theorem on partial differential equations with constant coefficients).

As a matter of fact, if $\Gamma_{K}$ is a primitive simple pseudogroup, the lifting theorem can be easily proved by using the results of V. Guillemin (see [2]).

If $\Gamma_{K}$ is primitive but not simple, the lifting theorem is true in the flat case ( $\Gamma_{M}$ and $\Gamma_{N}$ flat) but the demonstration requires, as in the affine case, Ehrenpreis-Malgrange's results. The method has been indicated in [1].

2. Construction of the counterexample. The counterexample is constructed by a refinement of the Lewy-Guillemin-Sternberg counterexample to the equivalence problem for $G$-structures [3].

(a) First, recall the data of this counterexample: We consider on $\mathbf{R}^{3}$ a transitive algebra of vector fields isomorphic to $g=S O(3, \mathrm{R}) .\left\{X_{1}, X_{2}, X_{3}\right\}$ is the standard basis of this algebra, that is to say, $X_{1}, X_{2}, X_{3}$ are globally defined vector fields on $\mathbf{R}^{3}$ satisfying the following relations:

$$
\left[X_{1}, X_{2}\right]=X_{3},\left[X_{1}, X_{3}\right]=X_{1},\left[X_{2}, X_{3}\right]=-X_{2} \text {. }
$$

Let $\left\{Y_{1}, Y_{2}\right\}$ be the standard basis of vector fields on $\mathrm{R}^{2} . \hat{E}$ is the $\hat{G}$ structure on $\mathrm{R}^{2} \times \mathrm{R}^{3}$ defined by the moving frame $\left\{Y_{1}, Y_{2}, X_{1}, X_{2}, X_{3}\right\}$, where $\hat{G}$ is the group of matrices of the form

$$
\left[\begin{array}{lllll}
1 & 0 & a & b & c \\
0 & 1 & d & e & f \\
0 & 0 & 1 & 0 & 0 \\
0 & 0 & 0 & 1 & 0 \\
0 & 0 & 0 & 0 & 1
\end{array}\right]
$$

$E$ is the $G$-structure on $\mathrm{R}^{2} \times \mathrm{R}^{3}$ defined by the same moving frame, where $G$ is the group of matrices of the form

$$
\left[\begin{array}{ccccc}
1 & 0 & a & b & c \\
0 & 1 & -b & a & d \\
0 & 0 & 1 & 0 & 0 \\
0 & 0 & 0 & 1 & 0 \\
0 & 0 & 0 & 0 & 1
\end{array}\right] .
$$


We denote by $\hat{\gamma}$ (resp. $\gamma$ ) the pseudogroup of automorphisms of $\hat{E}$ (resp. $E$ ) and by $\hat{l}$ (resp. $l$ ) the associated Lie algebra sheaf, in the terminology of [5].

$\hat{l}$ is a Lie algebra sheaf of vector fields of the following type:

$$
X=\xi+\phi_{1}\left(x_{1}, x_{2}, x_{3}\right) Y_{1}+\phi_{2}\left(x_{1}, x_{2}, x_{3}\right) Y_{2}
$$

where $\xi$ is in the algebra of (local) right-invariant vectorfields on $\mathbf{R}^{3} \subset \operatorname{SO}(3, \mathbf{R})$.

$X$ is in $l$ if and only if $\varphi_{1}, \varphi_{2}$ satisfy a system $\left(\Sigma_{l}\right)$ of linear partial differential equations.

(b) Let us consider now, for $p \geqslant 2$, the product $\mathbf{C}^{p} \times \mathbf{R}^{3}$ endowed with the $\widetilde{H}$-structure $\widetilde{E}$ obtained from the moving frame $\left\{\partial / \partial z_{1}, \ldots, \partial / \partial z_{p}, X_{1}, X_{2}\right.$, $\left.X_{3}\right\}$ by linear transformations in the group $\widetilde{H}$ of matrices of the following type:

$$
\left[\begin{array}{cccc}
\alpha_{j}^{i} & & & \times \times x \\
& & \times \times \times \\
0 \ldots 0 & 1 & 0 & 0 \\
0 \ldots & 0 & 1 & 0 \\
0 \ldots 0 & 0 & 0 & 1
\end{array}\right], \text { with }\left[\alpha_{j}^{i}\right] \in G L(p, \mathrm{C}) .
$$

Let $\widetilde{\Gamma}$ be the pseudogroup of automorphisms of this structure, $\widetilde{L}$ the associated $L A S$. We consider the projection $\tilde{\pi}: \widetilde{E} \rightarrow \mathbf{R}^{2} \times \mathbf{R}^{3}$ defined by:

$$
\tilde{\pi}(\tilde{z}(z, x))=\left(\operatorname{det}\left(\alpha_{j}^{i}\right), x\right) \text { where } \tilde{z}=\left\{\sum_{i} \alpha_{j}^{i} \frac{\partial}{\partial z_{j}}, \widetilde{X}_{1}(x), \widetilde{X}_{2}(x), \widetilde{X}_{3}(x)\right\}
$$

If $\tilde{Y}_{1}=\Sigma_{k=1}^{p} z_{k} \partial / \partial z_{k}$ and $\tilde{Y}_{2}=\Sigma_{k=1}^{p} i z_{k} \partial / \partial z_{k}$, let $\hat{\hat{L}}$ be the $L A S$ of vector fields on $\mathbf{C}^{p} \times \mathbf{R}^{3}$ of the following type:

$$
\tilde{X}=\sum_{k=1}^{p} X_{k}(x, z) \frac{\partial}{\partial z_{k}}+\varphi_{1}\left(x_{1}, x_{1}, x_{3}\right) \tilde{Y}_{1}+\varphi_{2}\left(x_{1}, x_{2}, x_{3}\right) \widetilde{Y}_{2}+\xi
$$

where $X_{k}$ is holomorphic with respect to $z$, with the sole condition $\Sigma_{k} \partial X_{k} / \partial z_{k}$ $=0$.

$\hat{L}$ defines a transitive Lie pseudogroup $\hat{\Gamma}$ on $\mathbf{C}^{p} \times \mathbf{R}^{3}$, with $\hat{\Gamma} \subset \tilde{\Gamma}$. By the projection $\tilde{\pi}, \hat{\Gamma}$ acting on $\widetilde{E}$ projects onto $\hat{\gamma}$. It is in this case a true quotient! The vector field (2) projects onto (1).

Let $\Gamma$ be the preimage of $\gamma$ by this projection $\hat{\Gamma} \rightarrow \hat{\gamma}$. The associated $L A S L$ is the $L A S$ of vector fields of the type (2) with the condition $\left(\Sigma_{l}\right)$ on $\varphi_{1}, \varphi_{2}$.

The fibration $\pi: \mathbf{C}^{p} \times \mathbf{R}^{3} \rightarrow \mathbf{R}^{3}$ allows us to consider $\Gamma$ as a primitive extension of $\operatorname{SO}(3, \mathbf{R})$. The counterexample follows now from the following proposition: 
Proposition. The equivalence theorem is not true for the pseudogroup $\Gamma$.

Proof. Let $\hat{E}^{1}$ be the second order structure associated to $\hat{\Gamma}$. We have a natural projection $\hat{\pi}^{1}: \hat{E}^{1} \rightarrow \hat{E}$. The second order structure $E^{1}$ associated to $\Gamma$ is the preimage of $E$ by $\hat{\pi}^{1}$.

If $E^{\prime}$ is an arbitrary $G$-subbundle of $\hat{E}$, let $E^{1 \prime}=\left[\hat{\pi}^{1}\right]^{-1}\left(E^{\prime}\right)$. By [3], $E^{\prime}$ defines an almost- $\gamma$-structure on $\mathbf{R}^{2} \times \mathbf{R}^{3}$. Then $E^{1 \prime}$ will define an almost- $\Gamma$ structure on $\mathbf{C}^{p} \times \mathbf{R}^{3}$. If $E^{\prime}$ is not a $\gamma$-structure, $E^{1 \prime}$ does not define a $\Gamma$. structure. Q. E. D.

3. The primitive lifting theorem in the flat case. Recall the principle of the demonstration in the flat case (following the method indicated in [1]): If $\Gamma_{K}$ is not simple, we consider the subpseudogroup $\Gamma_{K}^{\prime}$, the $L A S$ of which is the derived algebra of the $L A S$ of $\Gamma_{K}$. Then we can obtain a $\Gamma_{K}^{\prime}$-extension $\Gamma_{M}^{\prime}$ of $\Gamma_{N}$, with $\Gamma_{M}^{\prime} \subset \Gamma_{M}$

In the first order structure associated to $\Gamma_{M}$, we have an invariant foliation such that the first order structure associated to $\Gamma_{M}^{\prime}$ is foliated. If $\pi^{1}$ is the (local) projection onto a (local) quotient, we resolve the equivalence problem for the pseudogroup defined by passing to the quotient. This allows us to obtain for any almost- $\Gamma_{M}$-structure a subordinate almost- $\Gamma_{M}^{\prime}$-structure. Now, the primitive lifting theorem reduces to the simple case, which is elementary.

But the equivalence problem for the pseudogroup obtained by passing to the quotient by $\pi^{1}$ requires Ehrenpreis-Malgrange's theorem as an essential tool: in fact it is equivalent to the equivalence problem for a flat abelian pseudogroup.

\section{BIBLIOGRAPHY}

1. C. Buttin and P. Molino, Théorème général d'équivalence pour les pseudogroupes de Lie plats transitifs, J. Differential Geometry 9 (1974), 347-354. MR 50 \#5866.

2. V.W. Guillemin, A Jordan-Hölder decomposition for a certain class of infinite dimensional Lie algebras, J. Differential Geometry 2 (1968), 313-345. MR 41 \#8481.

3. V. W. Guillemin and S. Sternberg, The Lewy counterexample and the local equivalence problem for Gstructures, J. Differential Geometry 1 (1967), 127-131. MR 36 \#5850.

4. A. Pollack, The integrability problem for pseudogroup structures, J. Differential Geometry 9 (1974), 355-390.

5. I. M. Singer and S. Sternberg, The infinite groups of Lie and Cartan. I. The transitive groups, J. Analyse Math. 15 (1965), 1-114. MR 36 \#911.

INSTITUT DE MATHÉMATIQUES, UNIVERSITÉ DES SCIENCES ET TECHNIQUES DU LANGUEDOC, 34060 MONTPELLIER, FRANCE 